\title{
ON DONALDSON-THOMAS INVARIANTS OF THREEFOLD STACKS AND GERBES
}

\author{
AMIN GHOLAMPOUR AND HSIAN-HUA TSENG
}

(Communicated by Lev Borisov)

\begin{abstract}
We present a construction of Donaldson-Thomas invariants for three-dimensional projective Calabi-Yau Deligne-Mumford stacks. We also study the structure of these invariants for étale gerbes over such stacks.
\end{abstract}

\section{INTRODUCTION}

We work over the field of complex numbers throughout the paper. Let $\mathcal{X}$ be a smooth proper Deligne-Mumford (DM) stack with projective coarse moduli space $X$. Gromov-Witten (GW) theory, which roughly speaking concerns integrations against virtual fundamental classes of moduli spaces of twisted stable maps, is by now well-established [6], 1], 2] and has been an area of active research recently.

For 3-dimensional smooth projective varieties the so-called Donaldson-Thomas (DT) theory is constructed in [20]. An important special case is when DT theory concerns integration against virtual fundamental classes of the moduli spaces of torsion free, rank 1 sheaves with trivial determinants. It has been conjectured [14, 15, and proven in some cases, that this gives an equivalent theory to the GW theory of the ambient 3 -fold.

The first goal of this paper is to extend the construction of DT invariants to DM stacks. This is done in Section 2, Our construction is parallel to that of 20. and uses the moduli spaces of stable sheaves on a smooth projective DM stack $\mathcal{X}$ recently constructed by Nironi 17 . More precisely, we show that moduli spaces of stable sheaves on a 3 -dimensional DM stack $\mathcal{X}$ with trivial canonical bundle (i.e. Calabi-Yau) admit natural perfect obstruction theories, which we use to define invariants in case there are no strictly semistable sheaves.

For a given torsion element $[c] \in H_{e t}^{2}\left(\mathcal{X}, \mathbb{G}_{m}\right)$ represented by a 2-cocycle $c$, we will use the moduli space of stable $c$-twisted sheaves on $\mathcal{X}$ (see [5, 12, 17]). This is a connected component of the moduli space of sheaves on a $\mathbb{G}_{m}$-gerbe defined on $\mathcal{X}$ representing the element $[c]$ (see [17, Appendix A]). The perfect obstruction theory on the moduli space of stable sheaves (if it exists) induces a perfect obstruction theory on the moduli space of stable $c$-twisted sheaves.

As an application of the construction in this paper, we study the DT invariants of a $G$-gerbe $\mathcal{Y}$ over a 3 -dimensional Calabi-Yau stack $\mathcal{X}$, where $G$ is a finite group. This is the content of Section 3 . Our study is motivated by the physical conjecture in $[8$, which states that "conformal field theories on the $G$-gerbe $\mathcal{Y}$ are equivalent to

Received by the editors February 9, 2011 and, in revised form, May 12, 2011; June 1, 2011; and June 22, 2011.

2010 Mathematics Subject Classification. Primary 14N35.

(C) 2012 American Mathematical Society Reverts to public domain 28 years from publication 
conformal field theories on a dual space $\widehat{\mathcal{Y}}$ twisted by a B-field $c$." The construction of the dual space $\widehat{\mathcal{Y}}$ is explained in Definition 3.1.2. Mathematically, the B-field $c$ is a $\mathbb{G}_{m}$-valued cocycle on $\widehat{\mathcal{Y}}$. Its definition is explained in Section 3.1. The theme of Section 3 is a comparison between DT invariants of the gerbe $\mathcal{Y}$ and DT invariants of the dual $(\widehat{\mathcal{Y}}, c)$. Our Proposition 3.4 .3 can be interpreted as a DT-theoretic version of the physics conjecture.

In the presence of strictly semistable sheaves, moduli spaces of stable sheaves are not proper. In this situation it is desirable to extend the construction of generalized DT invariants [10] to our setting. We plan to pursue this elsewhere.

\section{DT INVARIANTS FOR DM-STACKS}

2.1. Review of Nironi's construction. In this section we construct a perfect obstruction theory for the moduli space of stable sheaves over a special class of 3-dimensional DM stacks that is used in this paper. The construction is similar to the case of smooth varieties (see [20]). This will allow us to define DT invariants for such stacks in cases where the moduli schemes are projective.

Let $\mathcal{X}$ be a DM stack with a projective moduli scheme $X$. We denote by c : $\mathcal{X} \rightarrow$ $X$ the "coarsening map" (i.e. the natural map from the stack $\mathcal{X}$ to its coarse moduli scheme $X$ ). We further assume that $\mathcal{X}$ is equipped with a generating sheaf $\mathcal{E}$ in the sense of [18, 17]. By definition $\mathcal{E}$ is a locally free sheaf on $\mathcal{X}$ whose fiber over any geometric point of $x \in \mathcal{X}$ contains the regular representation of the stabilizer group at $x$. Throughout the paper we fix a choice of a generating sheaf $\mathcal{E}$ of $\mathcal{X}$ and a polarization $\mathcal{O}_{X}(1)$ on $X$. Following [11] and [17, Definition 2.20], we call $\mathcal{X}$ projective if it satisfies these conditions 1

In 17. Nironi constructs the moduli space of semistable coherent sheaves on projective smooth DM stacks. We review part of his construction briefly and refer the reader to [17] for details. The main difference with the case of coherent sheaves on schemes is that the stability condition now depends on $\mathcal{E}$ as well as $\mathcal{O}_{X}(1)$. More precisely, for a pure coherent sheaf $\mathcal{F}$ on $\mathcal{X}$ stability is defined with respect to the Hilbert polynomial

$$
P_{\mathcal{F}}(m):=\chi\left(\mathcal{X}, \mathcal{F} \otimes \mathcal{E}^{\vee} \otimes \mathrm{c}^{*} \mathcal{O}_{X}(1)^{\otimes m}\right) .
$$

Let $p_{\mathcal{F}}$ be the monic polynomial obtained by dividing $P_{\mathcal{F}}$ by the coefficient of the leading term. $p_{\mathcal{F}}$ is the reduced Hilbert polynomial of $\mathcal{F}$. A pure coherent sheaf $\mathcal{F}$ on $\mathcal{X}$ is called semistable if $p_{\mathcal{F}^{\prime}} \leq p_{\mathcal{F}}$ for any proper subsheaf $\mathcal{F}^{\prime} \subset \mathcal{F}$. $\mathcal{F}$ is called stable if the inequality is always strict.

Let $P \in \mathbb{Q}[z]$, and let

$$
\mathcal{M}^{s}(\mathcal{X}, P)=\mathcal{M}^{s}\left(\mathcal{X}, \mathcal{E}, \mathcal{O}_{X}(1), P\right)
$$

be the moduli stack of pure stable sheaves $\mathcal{F}$ on $\mathcal{X}$ with $P_{\mathcal{F}}=P$. Nironi constructs $\mathcal{M}^{s}(\mathcal{X}, P)$ as a quotient stack $[Q / G L(N)]$, where $Q$ is an appropriate subscheme of a Quot scheme on $\mathcal{X}$ (see [18]). He shows that $\mathcal{M}^{s}(\mathcal{X}, P)$ is a $\mathbb{G}_{m}$-gerbe over a quasi-projective moduli scheme $M^{s}(\mathcal{X}, P)$. Moreover $M^{s}(\mathcal{X}, P)$ is shown to be a geometric quotient of $Q$, and GIT techniques provide a natural compactification of $M^{s}(\mathcal{X}, P)$, parameterizing the $S$-equivalence classes of semistable sheaves on $\mathcal{X}$ (see [17, Theorems 6.20-23]).

\footnotetext{
${ }^{1} \mathrm{~A}$ DM stack $\mathcal{X}$ is projective if and only if it is a tame separated global quotient with a projective moduli scheme (see [1] and [17, Theorem 2.21]).
} 
Let $\mathcal{L}$ be a line bundle on $\mathcal{X}$. Suppose that $\mathcal{M}^{s}(\mathcal{X}, P)$ is the moduli stack of torsion free stable sheaves with Hilbert polynomial $P$. In this case one can define

$$
\mathcal{M}^{s}(\mathcal{X}, P, \mathcal{L}) \subset \mathcal{M}^{s}(\mathcal{X}, P)
$$

as the moduli substack of torsion free stable sheaves with fixed determinant $\mathcal{L}$. We denote by $M^{s}(\mathcal{X}, P, \mathcal{L})$ the corresponding coarse moduli scheme. By the construction of Nironi and the discussion above, $\mathcal{M}^{s}(\mathcal{X}, P, \mathcal{L})$ is the fine moduli stack of DM type 2

2.2. Obstruction theory and DT invariants. By the following proposition there exist perfect obstruction theories on $\mathcal{M}^{s}(\mathcal{X}, P, \mathcal{L})$ and $M^{s}(\mathcal{X}, P)$ in the sense of [4. For the former moduli space, we implicitly assume that the objects are torsion free.

Proposition 2.2.1. Suppose $\mathcal{X}$ is a smooth projective DM stack of dimension 3 satisfying $\omega_{\mathcal{X}} \cong \mathcal{O}_{\mathcal{X}}$. Then there exist natural perfect obstruction theories on $\mathcal{M}^{s}(\mathcal{X}, P, \mathcal{L})$ and $M^{s}(X, P)$. Moreover, these obstruction theories are symmetric in the sense of $[3]$.

Proof. We first treat the case $\mathcal{M}_{\mathcal{L}}=\mathcal{M}^{s}(\mathcal{X}, P, \mathcal{L})$. Let

$$
\mathcal{U} \rightarrow \mathcal{X} \times \mathcal{M}_{\mathcal{L}}
$$

be the universal stable sheaf over $\mathcal{X} \times \mathcal{M}_{\mathcal{L}}$. For a closed point $m \in \mathcal{M}_{\mathcal{L}}$, let $\mathcal{U}_{m} \rightarrow \mathcal{X}$ be the stable sheaf parameterized by $m$. Note that we have the following equation on traceless Ext groups $\operatorname{Ext}_{\mathcal{X}}^{\bullet}(-,-)_{0}$ :

$$
\operatorname{Ext}_{\mathcal{X}}^{3}\left(\mathcal{U}_{m}, \mathcal{U}_{m}\right)_{0}=\operatorname{Ext}_{\mathcal{X}}^{0}\left(\mathcal{U}_{m}, \mathcal{U}_{m}\right)_{0}=0
$$

The first equality follows from Serre Duality for DM stacks (see [16, Theorem 1.32]) and the assumption that $\omega_{\mathcal{X}}$ is trivial. The second equality is because of the stability of $\mathcal{U}_{m}$.

The construction of the obstruction theory for $\mathcal{M}_{\mathcal{L}}$ is similar to the case of the moduli space of sheaves on smooth Calabi-Yau threefolds (see 20]). In what follows, $\mathbb{L}_{\square}$ denotes the cotangent complex of a DM stack (see [9]).

Let $\pi: \mathcal{X} \times \mathcal{M}_{\mathcal{L}} \rightarrow \mathcal{M}_{\mathcal{L}}$ be the projection. Composing the Atiyah class (see 9 , IV 2.3.6.2])

$$
\mathcal{U} \rightarrow \mathbb{L}_{\mathcal{X} \times \mathcal{M}_{\mathcal{L}}} \otimes \mathcal{U}[1]
$$

with the natural projection $\mathbb{L}_{\mathcal{X} \times \mathcal{M}_{\mathcal{L}}} \rightarrow \pi^{*} \mathbb{L}_{\mathcal{M}_{\mathcal{L}}}$ gives

$$
\mathcal{U} \rightarrow \pi^{*} \mathbb{L}_{\mathcal{M}_{\mathcal{L}}}[1] \otimes \mathcal{U}
$$

This gives a morphism

$$
R \mathcal{H o m}(\mathcal{U}, \mathcal{U}) \rightarrow \pi^{*} \mathbb{L}_{\mathcal{M}_{\mathcal{L}}}[1]
$$

Since $\pi$ is smooth of relative dimension 3, tensoring both sides by $\mathcal{O}[2]$ (note that $\left.\omega_{\pi}=\pi^{*} \omega_{\mathcal{X}} \cong \mathcal{O}\right)$ yields a morphism

$$
R \mathcal{H o m}(\mathcal{U}, \mathcal{U}[2]) \rightarrow \pi^{!} \mathbb{L}_{\mathcal{M}_{\mathcal{L}}}
$$

By the duality theorem (see [16, Corollary 1.22 and Theorem 1.32]) this gives a morphism

$$
R \pi_{*} R \mathcal{H o m}(\mathcal{U}, \mathcal{U}[2]) \rightarrow \mathbb{L}_{\mathcal{M}_{\mathcal{L}}}
$$

\footnotetext{
${ }^{2}$ In fact $\mathcal{M}^{s}(\mathcal{X}, P, \mathcal{L})$ is a $\mu_{r}$-gerbe over $M^{s}(\mathcal{X}, P, \mathcal{L})$, where $r$ is the rank of the objects parameterized by $\mathcal{M}^{s}(\mathcal{X}, P)$.
} 
which, after restricting the left hand side to its traceless part, gives a morphism

$$
\phi: \mathbb{E}=R \pi_{*} R \mathcal{H o m}(\mathcal{U}, \mathcal{U}[2])_{0} \rightarrow \mathbb{L}_{\mathcal{M}_{\mathcal{L}}} .
$$

In what follows we show that $(\mathbb{E}, \phi)$ is a perfect obstruction theory.

First note that $\mathbb{E}$ is perfect of perfect amplitude contained in $[-1,0]$. This is true because of (2.1) (see [21, Lemma 4.2]).

Next we need to show that $(\mathbb{E}, \phi)$ is an obstruction theory. Suppose $g: T \rightarrow$ $\mathcal{M}_{\mathcal{L}}$ is a morphism from a scheme $T$ and $T \rightarrow \bar{T}$ is an extension by a squarezero ideal $I$. Then we need to show that the obstruction to extending $g$ to $\bar{T}$ is a class $w \in \operatorname{Ext}_{T}^{1}\left(L g^{*} \mathbb{E}, I\right)$ obtained by composing $L g^{*} \phi$ with the natural maps $L g^{*} \mathbb{L}_{\mathcal{M}_{\mathcal{L}}} \rightarrow \mathbb{L}_{T} \rightarrow I[1]$. To show that $(\mathbb{E}, \phi)$ is an obstruction theory, it suffices to check the following criterion (see 4, Theorem 4.5]):

Claim. $w=0$ if and only if there exists an extension $\bar{g}: \bar{T} \rightarrow \mathcal{M}_{\mathcal{L}}$, and if it is nonempty, the set of all such $\bar{g}$ makes a torsor over $\operatorname{Ext}_{T}^{0}\left(L g^{*} \mathbb{E}, I\right)$.

We now prove this Claim. Let $f=(i d, g)$ and $\bar{f}=(i d, \bar{g})$. Consider the following diagram:

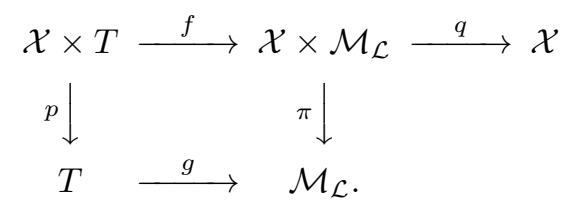

By standard arguments one obtains a natural identification

$$
\operatorname{Ext}_{T}^{i}\left(L g^{*} \mathbb{E}, I\right) \cong \operatorname{Ext}_{\mathcal{X} \times T}^{i+1}\left(f^{*} \mathcal{U}, f^{*} \mathcal{U} \otimes p^{*} I\right)_{0} .
$$

Because $\mathcal{M}_{\mathcal{L}}$ is a fine moduli space, deforming $g$ to $\bar{g}$ is equivalent to deforming $f^{*} \mathcal{U}$ to $\bar{f}^{*} \mathcal{U}$. The obstruction to the latter, denoted by $w^{\prime}$, is obtained by the composition

$$
f^{*} \mathcal{U} \rightarrow \mathbb{L}_{\mathcal{X} \times T} \otimes f^{*} \mathcal{U}[1] \rightarrow p^{*} I \otimes f^{*} \mathcal{U}[2]
$$

and then restricting it to the traceless part. The first map is the Atiyah class $a t\left(f^{*} \mathcal{U}\right)$, and the second one is induced from the natural map $\mathbb{L}_{\mathcal{X} \times T} \rightarrow p^{*} I[1]$. This is true because of [9, Proposition IV.3.1.8] and the fact that we have fixed the determinant. The reason for restricting it to the traceless part is that line bundles on $\mathcal{X}$ are unobstructed, and as in the case of sheaves on schemes (see [20]), one can show that the trace of the obstruction class of a sheaf $\mathcal{F}$ on a smooth DM stack is the obstruction class of $\operatorname{det}(\mathcal{F})$.

For a similar reason and by using (2.2), if $w^{\prime}=0$ the set of all deformations is a torsor over $\operatorname{Ext}_{T}^{0}\left(L g^{*} \mathbb{E}, I\right)$. So it remains to show that $w$ is mapped to $w^{\prime}$ under (2.2). We showed that $\phi: \mathbb{E} \rightarrow \mathbb{L}_{\mathcal{M}_{\mathcal{L}}}$ arises from the Atiyah class at $(\mathcal{U})$. By following exactly the same steps one can show that $L g^{*} \mathbb{E} \rightarrow L g^{*} \mathbb{L}_{\mathcal{M}_{\mathcal{L}}}$ arises from the Atiyah class at $\left(f^{*} \mathcal{U}\right)$. This means that the class $w$, which is the composition

$$
L g^{*} \mathbb{E} \stackrel{\phi}{\longrightarrow} L g^{*} \mathbb{L}_{\mathcal{M}_{\mathcal{L}}} \rightarrow \mathbb{L}_{T} \rightarrow I[1],
$$

gives rise to

$$
f^{*} \mathcal{U} \rightarrow \mathbb{L}_{\mathcal{X} \times T} \otimes f^{*} \mathcal{U}[1] \rightarrow p^{*} I \otimes f^{*} \mathcal{U}[2],
$$

which is what we need. This finishes the proof of the Claim and the construction of the perfect obstruction theory on $\mathcal{M}^{s}(\mathcal{X}, P, \mathcal{L})$.

To construct an obstruction theory on $M=M^{s}(\mathcal{X}, P)$, we just make the following modifications to the construction given above. First, $\mathcal{U}$ is replaced by the 
universal twisted sheaf on $M \times \mathcal{X}$ denoted by $U$ (see [5, Section 3.3]). Second, the natural candidate

$$
R \pi_{*} R \mathcal{H o m}(U, U[2]) \cong\left(R \pi_{*} R \mathcal{H o m}(U, U)\right)^{\vee}[-1]
$$

for the obstruction theory is not perfect, where now $\pi: \mathcal{X} \times M \rightarrow M$ is the projection. However, repeating the arguments in [21, Section 4.4], it can be shown that the trimmed complex

$$
\left(\tau^{[1,2]} R \pi_{*} R \mathcal{H o m}(U, U)\right)^{\vee}[-1]
$$

gives rise to a perfect obstruction theory on $M^{s}(\mathcal{X}, P)$.

The symmetry of the obstruction theories on $\mathcal{M}^{s}(\mathcal{X}, P, \mathcal{L})$ and $M^{s}(\mathcal{X}, P)$ follows easily from Serre duality and the Calabi-Yau condition $\omega_{\mathcal{X}} \cong \mathcal{O}_{\mathcal{X}}$.

If $\mathcal{X}$ is as in Proposition 2.2.1, then by the symmetry property of the obstruction theories the expected dimensions of $\mathcal{M}^{s}(\mathcal{X}, P, \mathcal{L})$ and $M^{s}(\mathcal{X}, P)$ are 0 . By Proposition 2.2.1 and [4] we have

Proposition 2.2.2. Suppose $\mathcal{X}$ is a smooth projective DM stack of dimension 3 satisfying $\omega_{\mathcal{X}} \cong \mathcal{O}_{\mathcal{X}}$. Then $\mathcal{M}^{s}(\mathcal{X}, P, \mathcal{L})$ and $M^{s}(\mathcal{X}, P)$ carry virtual 0 -cycles denoted by $\left[\mathcal{M}^{s}(\mathcal{X}, P, \mathcal{L})\right]^{\text {vir }}$ and $\left[M^{s}(\mathcal{X}, P)\right]^{\text {vir }}$.

Let $\nu_{\mathcal{M}^{s}}$ (respectively, $\nu_{\mathcal{M}_{\mathcal{L}}^{s}}$ ) be the Behrend's function ([3, 10]) defined on $\mathcal{M}^{s}(\mathcal{X}, P)$ (respectively, $\left.\mathcal{M}^{s}(\mathcal{X}, P, \mathcal{L})\right)$. Then we define the corresponding DT invariants as follows.

Definition 2.2.3. Let $\mathcal{X}$ be as in Proposition 2.2.1 $P \in \mathbb{Q}[z]$, and $\mathcal{L}$ be a line bundle on $\mathcal{X}$. Then we define the Donaldson-Thomas invariant 3 of $\mathcal{X}$ corresponding to $P($ and $\mathcal{L})$ as the weighted Euler characteristics

$$
\begin{gathered}
\operatorname{DT}(\mathcal{X}, P)=-\chi^{n a}\left(\mathcal{M}^{s}(\mathcal{X}, P), \nu_{\mathcal{M}^{s}}\right), \\
\operatorname{DT}(\mathcal{X}, P, \mathcal{L})=\chi\left(\mathcal{M}^{s}(\mathcal{X}, P, \mathcal{L}), \nu_{\mathcal{M}_{\mathcal{L}}^{s}}\right) .
\end{gathered}
$$

Here $\chi^{n a}$ is the naïve Euler characteristic defined for Artin stacks (see 10, Definition 2.3]) and $\chi$ denotes the Euler characteristic of DM stacks.

Remark 2.2.1.

(1) Let $r$ be the rank of the objects parameterized by $\mathcal{M}^{s}(\mathcal{X}, P)$, and let $\nu_{M^{s}}$ (respectively, $\nu_{M_{\mathcal{L}}^{s}}$ ) be the Behrend's function for $M^{s}(\mathcal{X}, P)$ (respectively, for $\left.M^{s}(\mathcal{X}, P, \mathcal{L})\right)$. Then by the properties of the Behrend's function and weighted Euler characteristic (see [3, 10]), we have

$$
\operatorname{DT}(\mathcal{X}, P)=\chi\left(M^{s}(\mathcal{X}, P), \nu_{M^{s}}\right)
$$

and

$$
\operatorname{DT}(\mathcal{X}, P, \mathcal{L})=\frac{1}{r} \chi\left(M^{s}(\mathcal{X}, P, \mathcal{L}), \nu_{M_{\mathcal{L}}^{s}}\right) .
$$

The reason for the first equality is that the natural coarsening map $c_{\mathcal{M}^{s}}$ : $\mathcal{M}^{s}(\mathcal{X}, P) \rightarrow M^{s}(\mathcal{X}, P)$ is smooth of relative dimension -1 and $\operatorname{so} c_{\mathcal{M}^{s}}^{*}\left(\nu_{M^{s}}\right)$ $=-\nu_{\mathcal{M}}$.

\footnotetext{
${ }^{3}$ These invariants depend on the choices of $\mathcal{E}$ and $\mathcal{O}_{X}(1)$; however, this dependence is suppressed in our notation.
} 
(2) Suppose that there are no strictly semistable sheaves $\mathcal{F}$ on $\mathcal{X}$ satisfying $P_{\mathcal{F}}=P$. It is known [17] that in this case $M^{s}(\mathcal{X}, P)$ and $\mathcal{M}^{s}(\mathcal{X}, P, \mathcal{L})$ are proper, and hence the virtual classes $\left[M^{s}(\mathcal{X}, P)\right]^{\text {vir }}$ and $\left[\mathcal{M}^{s}(\mathcal{X}, P, \mathcal{L})\right]^{\text {vir }}$ can be integrated. By [3, Theorem 4.18] and [10, Remark 5.14]

$$
\operatorname{DT}(\mathcal{X}, P, \mathcal{L})=\operatorname{deg}\left(\left[\mathcal{M}^{s}(\mathcal{X}, P, \mathcal{L})\right]^{\text {vir }}\right)
$$

and

$$
\operatorname{DT}(\mathcal{X}, P)=\operatorname{deg}\left(\left[M^{s}(\mathcal{X}, P)\right]^{\text {vir }}\right) .
$$

(3) If there are strictly semistable sheaves, then $\operatorname{DT}(\mathcal{X}, P)$ and $\operatorname{DT}(\mathcal{X}, P, \mathcal{L})$ are not in general deformation invariant. One way to fix this is to extend the construction of generalized Donaldson-Thomas invariants [10] to this setting. This will be explored elsewhere.

\section{A DECOMPosition RESUlt FOR DT INVARIANTS ON GERBES}

The purpose of this section is to study DT invariants of étale gerbes.

3.1. Étale gerbes. We begin with a review of some basic notions of étale gerbes and the construction of their duals. Let $G$ be a finite group 4 Let $\mathcal{X}$ be a smooth projective Deligne-Mumford stack with coarse moduli scheme $X$. Let $B G$ denote the stack of $G$-torsors.

Definition 3.1.1 (see e.g. [7, Definition 3.1]). A $G$-gerbe over $\mathcal{X}$ is a DeligneMumford stack $\mathcal{Y}$ together with a morphism $\mathcal{Y} \rightarrow \mathcal{X}$ such that there exists a faithfully flat, locally of finite presentation map $X^{\prime} \rightarrow \mathcal{X}$ such that $\mathcal{Y} \times \mathcal{X} X^{\prime} \simeq$ $B G \times X^{\prime}$.

In this way one can view $B G$ as a $G$-gerbe over a point.

Let $\operatorname{Out}(G)$ denote the group of outer automorphisms of $G$. By definition, $\operatorname{Out}(G)$ is the quotient of the group $\operatorname{Aut}(G)$ of automorphisms of $G$ by the normal subgroup $\operatorname{Inn}(G)$ of inner automorphisms of $G$,

$$
\operatorname{Out}(G)=\operatorname{Aut}(G) / \operatorname{Inn}(G) .
$$

Given a $G$-gerbe $\mathcal{Y} \rightarrow \mathcal{X}$, there is a naturally defined $O u t(G)$-bundle $\overline{\mathcal{Y}} \rightarrow \mathcal{X}$, called the band. See [7, Definition 3.3] for a detailed definition. We say that the $G$-gerbe $\mathcal{Y} \rightarrow \mathcal{X}$ has a trivial band if the $\operatorname{Out}(G)$-bundle $\overline{\mathcal{Y}} \rightarrow \mathcal{X}$ is endowed with a section (hence is trivialized by this section).

Let $\widehat{G}$ denote the set of isomorphism classes of irreducible representations of $G$. Note that $\widehat{G}$ is a finite set and that the cardinality of $\widehat{G}$ coincides with the number of conjugacy classes of $G$. We may also view $\widehat{G}$ as a disjoint union of points.

Let $\rho: G \rightarrow \operatorname{End}\left(V_{\rho}\right)$ be an irreducible representation of $G$, and let $\phi \in \operatorname{Aut}(G)$. Then the composite

$$
\rho \circ \phi^{-1}: G \rightarrow \operatorname{End}\left(V_{\rho}\right)
$$

is an irreducible representation of $G$. It is easy to see that this induces an action of $\operatorname{Out}(G)$ on $\widehat{G}$. Note that the isomorphism class $\left[1_{t r}\right]$ of the 1-dimensional trivial representation of $G$ is fixed by this $\operatorname{Out}(G)$ action.

\footnotetext{
${ }^{4} G$ is viewed as a finite group scheme over Spec $\mathbb{C}$.
} 
Definition 3.1.2 (see [8, page 760], [19, equation (1.3)]). Define

$$
\widehat{\mathcal{Y}}:=[\overline{\mathcal{Y}} \times \widehat{G} / \text { Out }(G)] .
$$

There is a natural map $\widehat{\mathcal{Y}} \rightarrow \mathcal{X}$ induced from the map $\overline{\mathcal{Y}} \rightarrow \mathcal{X}$.

Remark 3.1.3.

(1) The morphism $\widehat{\mathcal{Y}} \rightarrow \mathcal{X}$ is finite and étale. The stack $\widehat{\mathcal{Y}}$ is disconnected.

(2) If the $G$-gerbe $\widehat{\mathcal{Y}} \rightarrow \mathcal{X}$ has trivial band, then $\widehat{\mathcal{Y}}$ is a disjoint union of several copies of $\mathcal{X}$, and the map $\widehat{\mathcal{Y}} \rightarrow \mathcal{X}$ restricts to the identity on each copy.

For each isomorphism class $[\rho] \in \widehat{G}$ we fix a representation $\rho: G \rightarrow \operatorname{End}\left(V_{\rho}\right)$ in this class. To each $(x,[\rho]) \in \widehat{\mathcal{Y}}$ we assign the vector space $V_{\rho}$. This defines a family of vector spaces over $\widehat{\mathcal{Y}}$, which is in general not a vector bundle over $\widehat{\mathcal{Y}}$. The obstruction to find a vector bundle over $\widehat{\mathcal{Y}}$ with fiber over $(x,[\rho])$ being $V_{\rho}$ is a $\mathbb{G}_{m}$-valued 2-cocycle on $\widehat{\mathcal{Y}}$ whose inverse is denoted by $c$. More discussions on $c$ can be found in [8, Sections 4.1-4.2].

As observed in [8, Section 9.5], the cocycle $c$ is locally constant and $c$ represents a torsion class in the cohomology $H_{e t}^{2}\left(\widehat{\mathcal{Y}}, \mathbb{G}_{m}\right)$.

Another way to understand the cocycle $c$ is the following. The failure for $V_{\rho}$ 's to form a vector bundle is due to the fact that they glue up to scalars. In other words $V_{\rho}$ 's glue to a twisted sheaf (see [5] for definitions). This twisted sheaf is equivalent (see [12, Section 2.1.3]) to a sheaf on a $\mathbb{G}_{m}$-gerbe over $\widehat{\mathcal{Y}}$. This $\mathbb{G}_{m}$-gerbe turns out to be flat, and the inverse of its class, which is an element in $H_{\text {et }}^{2}\left(\widehat{\mathcal{Y}}, \mathbb{G}_{m}\right)$, is represented by the 2-cocycle $c$.

3.2. Equivalence. In what follows we will be concerned with sheaves on gerbes and a decomposition statement about DT invariants for Calabi-Yau gerbes, which was inspired by 8 .

We continue to use the notation in the previous section. Let $\mathcal{Y} \rightarrow \mathcal{X}$ be a $G$ gerbe over a smooth projective DM stack $\mathcal{X}$ and let $\widehat{\mathcal{Y}} \rightarrow \mathcal{X}$ and $c$ be as constructed before. Note that $\mathcal{Y}$ is also a smooth projective DM stack, and the coarse moduli space of $\mathcal{Y}$ is $X$. Let $c_{\mathcal{Y}}: \mathcal{Y} \rightarrow X$ be the coarsening map. By construction, $\widehat{\mathcal{Y}}$ is also smooth and projective. Let $c_{\widehat{\mathcal{Y}}}: \widehat{\mathcal{Y}} \rightarrow \widehat{Y}$ denote its coarsening map, and let $\pi_{\widehat{Y}}: \widehat{Y} \rightarrow X$ be the map between coarse moduli spaces induced by $\widehat{\mathcal{Y}} \rightarrow \mathcal{X}$.

Fix an ample line bundle $\mathcal{O}_{X}(1)$ of $X$. Note that the pull-back $\pi_{\widehat{Y}}^{*} \mathcal{O}_{X}(1)$ is an ample line bundle of $\widehat{Y}$.

Let $\operatorname{Coh}(\mathcal{Y})$ denote the abelian category of coherent sheaves on $\mathcal{Y}$ and $\operatorname{Coh}(\widehat{\mathcal{Y}}, c)$ denote the abelian category of coherent $c$-twisted sheaves on $\widehat{\mathcal{Y}}$. We refer to [5] and [12] for detailed discussions on the theory of twisted sheaves. The following result is proven in [19].

Theorem 3.2.1 (see [19, Section 7]). There is natural functor

$$
F: \operatorname{Coh}(\mathcal{Y}) \rightarrow \operatorname{Coh}(\widehat{\mathcal{Y}}, c),
$$

which is an equivalence of abelian categories.

The construction of this functor $F$ is rather involved. Details can be found in [19, Section 7]. Roughly speaking, the inverse functor $\operatorname{Coh}(\widehat{\mathcal{Y}}, c) \rightarrow \operatorname{Coh}(\mathcal{Y})$ can be 
understood as taking $(-) \otimes \mathcal{V}_{\rho}$, where $\mathcal{V}_{\rho}$ is the aforementioned $c^{-1}$-twisted sheaf with fibers $V_{\rho}$.

As noted above, $\widehat{\mathcal{Y}}$ is disconnected. Let $\widehat{\mathcal{Y}}=\coprod_{i \in \mathcal{I}} \widehat{\mathcal{Y}}_{i}$ be the decomposition of $\widehat{\mathcal{Y}}$ into connected components, and let $c_{i}$ be the 2-cocycle on $\widehat{\mathcal{Y}}_{i}$ obtained by the restriction of $c$. By definition, we have

$$
\operatorname{Coh}(\widehat{\mathcal{Y}}, c)=\bigoplus_{i \in \mathcal{I}} \operatorname{Coh}\left(\widehat{\mathcal{Y}}_{i}, c_{i}\right)
$$

Consequently there is a decomposition of $K$-groups

$$
K(\operatorname{Coh}(\widehat{\mathcal{Y}}, c))=\bigoplus_{i \in \mathcal{I}} K\left(\operatorname{Coh}\left(\widehat{\mathcal{Y}}_{i}, c_{i}\right)\right) .
$$

On the other hand, $K(\mathcal{Y})=K(\operatorname{Coh}(\mathcal{Y}))=K(\operatorname{Coh}(\widehat{\mathcal{Y}}, c))$. Therefore we get a decomposition of $K(\mathcal{Y})$ :

$$
K(\mathcal{Y})=\bigoplus_{i \in \mathcal{I}} K_{i}, \quad K_{i}:=K\left(\operatorname{Coh}\left(\widehat{\mathcal{Y}}_{i}, c_{i}\right)\right)
$$

Given $\mathcal{F} \in \operatorname{Coh}(\mathcal{Y})$, we write

$$
F(\mathcal{F})=\bigoplus_{i \in \mathcal{I}} F(\mathcal{F})_{i}, \quad F(\mathcal{F})_{i} \in \operatorname{Coh}\left(\widehat{\mathcal{Y}}_{i}, c_{i}\right) .
$$

Since (3.1) is an equivalence of abelian categories, it preserves exact sequences. Hence for $\mathcal{F} \in \operatorname{Coh}(\mathcal{Y})$ and a subsheaf $\mathcal{F}^{\prime} \subset \mathcal{F}$, the components $F\left(\mathcal{F}^{\prime}\right)_{i}$ are subsheaves of $F(\mathcal{F})_{i}$. Also for $\mathcal{F}_{1}, \mathcal{F}_{2} \in C o h(\mathcal{Y})$ we have the equality on Hom spaces:

$$
\operatorname{Hom}_{\operatorname{Coh}(\mathcal{Y})}\left(\mathcal{F}_{1}, \mathcal{F}_{2}\right)=\bigoplus_{i \in \mathcal{I}} \operatorname{Hom}_{\operatorname{Coh}\left(\widehat{\mathcal{Y}}_{i}, c_{i}\right)}\left(F\left(\mathcal{F}_{1}\right)_{i}, F\left(\mathcal{F}_{2}\right)_{i}\right) .
$$

By the construction of the equivalence (3.1), it is easy to check that if $\mathcal{V}$ is a generating sheaf of $\mathcal{Y}$, then $F(\mathcal{V})$ is a generating $c$-twisted sheaf of $\widehat{\mathcal{Y}}$. Hence $F(\mathcal{V})_{i} \in \operatorname{Coh}\left(\widehat{\mathcal{Y}}_{i}, c_{i}\right)$ is a generating $c_{i}$-twisted sheaf of $\widehat{\mathcal{Y}}_{i}$. Since $G$ acts trivially on $c_{\mathcal{Y}}^{*} \mathcal{O}_{X}(1)$, the construction of the equivalence (3.1) implies that

$$
F\left(\mathcal{F} \otimes c_{\mathcal{Y}}^{*} \mathcal{O}_{X}(1)^{\otimes m}\right)=F(\mathcal{F}) \otimes c_{\widehat{\mathcal{Y}}}^{*} \pi_{\widehat{Y}}^{*} \mathcal{O}_{X}(1)^{\otimes m} .
$$

From now on, fix a generating sheaf $\mathcal{E}$ on $\mathcal{X}$ and an ample line bundle $\mathcal{O}_{X}(1)$ on $X$. Also fix the generating $c$-twisted sheaf $F(\mathcal{E})$ on $\widehat{\mathcal{Y}}$ and an ample line bundle $\pi_{\widehat{Y}}^{*} \mathcal{O}_{X}(1)$ on $\widehat{Y}$. With these choices it follows that the Hilbert polynomial $P_{\mathcal{F}}$ of $\mathcal{F}$ coincides with the Hilbert polynomial $P_{F(\mathcal{F})}$ of $F(\mathcal{F})$. More precisely,

$$
P_{\mathcal{F}}=P_{F(\mathcal{F})}=\sum_{i \in \mathcal{I}} P_{F(\mathcal{F})_{i}}
$$

\subsection{Invariants. Let}

$$
C(\mathcal{Y})=\{[\mathcal{F}] \in K(\mathcal{Y}) \mid 0 \neq \mathcal{F} \in C o h(\mathcal{Y})\}
$$

be the positive cone in $K(\mathcal{Y})$. Then $C(\mathcal{Y})=\bigoplus_{i \in \mathcal{I}} C_{i}$, corresponding to the decomposition (3.2). Let $k \in C(\mathcal{Y})$, and let $\mathcal{M}^{s}(\mathcal{Y}, k)$ be the moduli stack of pure stable sheaves on $\mathcal{Y}$ of class $k$. It is evident that $\mathcal{M}^{s}(\mathcal{Y}, k)$ is a component of the moduli stack of pure stable sheaves with fixed Hilbert polynomials. Suppose further that 
$k \in C_{i}$ in the decomposition above. Note that for any $\mathcal{F} \in \operatorname{Coh}(\mathcal{Y})$ of class $k$, we have $F(\mathcal{F})=F(\mathcal{F})_{i} \in \operatorname{Coh}\left(\widehat{\mathcal{Y}}, c_{i}\right)$, namely

$$
F(\mathcal{F})_{j}=0 \text { for } j \neq i .
$$

By (3.4) and (3.5) we have the following relations between (reduced) Hilbert polynomials:

$$
P_{\mathcal{F}}=P_{F(\mathcal{F})_{i}}, \quad p_{\mathcal{F}}=p_{F(\mathcal{F})_{i}} .
$$

Consequently $\mathcal{F}$ is stable if and only if $F(\mathcal{F})_{i}$ is stable.

Therefore the equivalence (3.1) yields a set-theoretic bijection

$$
\mathcal{M}^{s}(\mathcal{Y}, k) \rightarrow \mathcal{M}^{s}\left(\left(\widehat{\mathcal{Y}}_{i}, c_{i}\right), k\right), \quad[\mathcal{F}] \mapsto\left[F(\mathcal{F})_{i}\right]
$$

Here $\mathcal{M}^{s}\left(\left(\widehat{\mathcal{Y}}_{i}, c_{i}\right), k\right)$ denotes the moduli of stable $c_{i}$-twisted sheaves on $\widehat{\mathcal{Y}}_{i}$ of class $k$. As mentioned in Section 1, $\mathcal{M}^{s}\left(\left(\widehat{\mathcal{Y}}_{i}, c_{i}\right), k\right)$ is realized as a connected component of the certain moduli space of sheaves on a $\mu_{N}$-gerbe over $\widehat{\mathcal{Y}}_{i}$ for $N \gg 0$, and hence Nironi's construction applies (see [17, Appendix A]).

Proposition 3.3.1. There is an isomorphism of stacks

$$
\mathcal{M}^{s}(\mathcal{Y}, k) \simeq \mathcal{M}^{s}\left(\left(\widehat{\mathcal{Y}}_{i}, c_{i}\right), k\right) .
$$

Proof. One may prove this by checking that deformation theory on both sides agree to all order, using (3.3). We will pursue a different way to construct the isomorphism, as follows. Clearly the product $\mathcal{M}^{s}(\mathcal{Y}, k) \times \mathcal{Y}$ is a $G$-gerbe over $\mathcal{M}^{s}(\mathcal{Y}, k) \times \mathcal{X}$. By construction we see that the dual of this $G$-gerbe is

$$
\mathcal{M}^{s} \widehat{\mathcal{Y}, k)} \times \mathcal{Y}=\mathcal{M}^{s}(\mathcal{Y}, k) \times \widehat{\mathcal{Y}} .
$$

Moreover the 2-cocycle in this case is the pull-back of $c$ on $\widehat{\mathcal{Y}}$ via the projection $\mathcal{M}^{s}(\mathcal{Y}, k) \times \widehat{\mathcal{Y}} \rightarrow \widehat{\mathcal{Y}}$. Let $\mathcal{U} \rightarrow \mathcal{M}^{s}(\mathcal{Y}, k) \times(\mathcal{Y})$ be the universal stable sheaf. Note that there exists an equivalence (3.1) for any $G$-gerbe. Applying such an equivalence to the sheaf $\mathcal{U}$ over the $G$-gerbe $\mathcal{M}^{s}(\mathcal{Y}, k) \times \mathcal{Y}$, we obtain a twisted sheaf $F(\mathcal{U})$ over $\mathcal{M}^{s}(\mathcal{Y}, k) \times \widehat{\mathcal{Y}}$. It is easy to check that $F(\mathcal{U})$ is a family over $\mathcal{M}^{s}(\mathcal{Y}, k)$ of $c_{i}$-twisted stable sheaves with class $k$. This defines a morphism

$$
\phi: \mathcal{M}^{s}(\mathcal{Y}, k) \rightarrow \mathcal{M}^{s}\left(\left(\widehat{\mathcal{Y}}_{i}, c_{i}\right), k\right) .
$$

To show that $\phi$ is an isomorphism, we may show that $\phi$ induces an isomorphism between the functors of points of $\mathcal{M}^{s}(\mathcal{Y}, k)$ and $\mathcal{M}^{s}\left(\left(\widehat{\mathcal{Y}}_{i}, c_{i}\right), k\right)$. At the level of objects, we need to show two things.

(1) Let $S$ be a scheme. Let $\varphi_{1}: S \rightarrow \mathcal{M}^{s}(\mathcal{Y}, k)$ and $\varphi_{2}: S \rightarrow \mathcal{M}^{s}(\mathcal{Y}, k)$ be morphisms such that $\phi \circ \varphi_{1}=\phi \circ \varphi_{2}$. Then $\varphi_{1}=\varphi_{2}$. To see this, for $i=1,2$ let $\mathcal{U}_{i} \rightarrow S \times \mathcal{Y}$ be the stable sheaf corresponding to $\varphi_{i}$. Then the twisted sheaf corresponding to $\phi \circ \varphi_{i}$ is $F\left(\mathcal{U}_{i}\right) \rightarrow S \times \widehat{\mathcal{Y}}$. Then $\phi \circ \varphi_{1}=\phi \circ \varphi_{2}$ means $F\left(\mathcal{U}_{1}\right)=F\left(\mathcal{U}_{2}\right)$. Applying the inverse functor of $F$, we get $\mathcal{U}_{1}=\mathcal{U}_{2}$ as sheaves on $S \times \mathcal{Y}$, which means that $\varphi_{1}=\varphi_{2}$.

(2) Let $S$ be a scheme. Let $\varphi: S \rightarrow \mathcal{M}^{s}\left(\left(\widehat{\mathcal{Y}}_{i}, c_{i}\right), k\right)$ be a morphism. Then there exists a morphism $\psi: S \rightarrow \mathcal{M}^{s}(\mathcal{Y}, k)$ such that $\phi \circ \psi=\varphi$. To see this, let $\mathcal{U}_{\varphi}^{t w} \rightarrow S \times \widehat{\mathcal{Y}}$ be the twisted sheaf corresponding to $\varphi$. Applying the inverse functor of $F$ yields a sheaf $F^{-1}\left(\mathcal{U}_{\varphi}^{t w}\right) \rightarrow S \times \mathcal{Y}$. This gives a morphism $\psi: S \rightarrow \mathcal{M}^{s}(\mathcal{Y}, k)$. By construction, the twisted sheaf corresponding to $\phi \circ \psi$ is $F\left(F^{-1}\left(\mathcal{U}_{\varphi}^{t w}\right)\right)=\mathcal{U}_{\varphi}^{t w} \rightarrow S \times \widehat{\mathcal{Y}}$. This shows that $\phi \circ \psi=\varphi$. 
The argument at the level of morphisms is completely analogous and is left to the reader.

Now suppose in addition that $\mathcal{X}$ is a Calabi-Yau Deligne-Mumford stack of dimension 3. Hence both $\mathcal{Y}$ and $\widehat{\mathcal{Y}}$ are Calabi-Yau of dimension 3. Then we can define DT invariants $\operatorname{DT}(\mathcal{Y}, k)$ and $\operatorname{DT}\left(\left(\widehat{\mathcal{Y}}_{i}, c_{i}\right), k_{i}\right)$ as in Section 3.3 and by Proposition 3.3 .1 we have

\section{Proposition 3.3.2.}

$$
\operatorname{DT}(\mathcal{Y}, k)=\operatorname{DT}\left(\left(\widehat{\mathcal{Y}}_{i}, c_{i}\right), k\right) .
$$

Remark 3.3.3. One can see directly, using (3.3), that the 2-term perfect obstruction theory associated to $\mathcal{M}^{s}(\mathcal{Y}, k)$ is mapped to the one on $\mathcal{M}^{s}\left(\left(\widehat{\mathcal{Y}}_{i}, c_{i}\right), k\right)$. This gives an alternative proof for the proposition above in cases where $k \in C(\mathcal{Y})$ is such that for sheaves of class $k$ semistability and stability coincide.

3.4. Decomposition. Using the notation in Sections 3.1 3.2, we let $\mathcal{Y}$ be a $G$ gerbe over a smooth projective DM stack $\mathcal{X}$, and let $k \in C(\mathcal{Y})$. Suppose $k_{i}$ is the $C_{i}$-component of $k$ in the decomposition $C(\mathcal{Y})=\bigoplus_{i \in \mathcal{I}} C_{i}$ induced from (3.2),

$$
k=\sum_{i} k_{i}, \quad k_{i} \in C_{i}
$$

Let $\mathcal{M}(\mathcal{Y}, k)$ be the moduli stack 5 of coherent sheaves on $\mathcal{Y}$ of class $k$, and let $\mathcal{M}\left(\left(\widehat{\mathcal{Y}}_{i}, c_{i}\right), k_{i}\right)$ be the moduli stack of $c_{i}$-twisted coherent sheaves on $\widehat{\mathcal{Y}}_{i}$ of class $k_{i}$.

\section{Proposition 3.4.1.}

(1) There is an isomorphism of stacks,

$$
\mathcal{M}(\mathcal{Y}, k) \simeq \prod_{i \in \mathcal{I}} \mathcal{M}\left(\left(\widehat{\mathcal{Y}}_{i}, c_{i}\right), k_{i}\right)
$$

(2) Moreover if $\mathcal{M}^{s}(\mathcal{Y}, k)$ is nonempty, then there exists a unique $j \in \mathcal{I}$ such that $k_{i}=0$ for $i \neq j$, and in this case the isomorphism above restricts to the isomorphism

$$
\mathcal{M}^{s}(\mathcal{Y}, k) \simeq \mathcal{M}^{s}\left(\left(\widehat{\mathcal{Y}}_{j}, c_{j}\right), k_{j}\right) .
$$

Proof.

(1) This is proved by the same arguments as in the proof of Proposition 3.3.1 by noting that the equivalence functor $F$ maps $\mathcal{M}(\mathcal{Y}, k)$ to the moduli stack of $c$-twisted sheaves on $\widehat{\mathcal{Y}}$, and the latter moduli stack can be expressed as the product of the moduli stacks on the connected components of $\widehat{\mathcal{Y}}$.

(2) Note that if there is a stable sheaf $\mathcal{F}$ on $\mathcal{Y}$ in class $k$, then $\mathcal{F}$ is in particular simple, i.e. $\operatorname{End}(\mathcal{F}) \cong \mathbb{C}$. Since the functor $F$ is an equivalence, we have $\operatorname{End}(\mathcal{F}) \cong \operatorname{End}(F(\mathcal{F}))$. This implies that $\operatorname{End}(F(\mathcal{F})) \cong \mathbb{C}$. But this is only possible if $F(\mathcal{F})_{i}=0$ for all $i \in \mathcal{I}$ except one that we denote by $j$. Together with (3.4) this shows that $\mathcal{F}$ is stable if and only if $F(\mathcal{F})=F(\mathcal{F})_{j}$ is stable. The rest of the proof is exactly the same as the proof of Proposition 3.3.1.

\footnotetext{
${ }^{5}$ Note that no stability is imposed here.
} 
Remark 3.4.2. Stability of sheaves on the right hand side of (3.6) does not necessarily imply the stability of sheaves on the left hand side. For example, suppose that for any $i \in \mathcal{I}$ the pair $\left(\widehat{\mathcal{Y}}_{i}, c_{i}\right)$, viewed as a cyclic gerbe, is optimal in the sense of 12 , Definition 2.2.5.2], and consider the classes $k_{i}$ which consist of $c_{i}$-twisted sheaves of minimal ranks (in the sense of [12, Definition 2.2.5.1]). Then the twisted sheaves of classes $k_{i}$ are stable by [13, Lemma 3.2.1.8]. But the corresponding coherent sheaf in $\mathcal{M}(\mathcal{Y}, k)$ is not necessarily stable.

The following is our decomposition statement.

Proposition 3.4.3. In the situation of Proposition 3.4.1 we have

$$
\chi^{n a}\left(\mathcal{M}(\mathcal{Y}, k), \nu_{\mathcal{M}}\right)=\prod_{i \in \mathcal{I}} \chi^{n a}\left(\mathcal{M}\left(\left(\widehat{\mathcal{Y}}_{i}, c_{i}\right), k_{i}\right), \nu_{\mathcal{M}_{i}}\right),
$$

where $\nu_{\mathcal{M}}$ and $\nu_{\mathcal{M}_{i}}$ are the Behrend's functions of $\mathcal{M}(\mathcal{Y}, k)$ and $\mathcal{M}\left(\left(\widehat{\mathcal{Y}}_{i},, c_{i}\right), k_{i}\right)$, respectively. Moreover suppose that $\mathcal{X}$ is a Calabi-Yau DM stack of dimension 3 and $\mathcal{M}^{s}(\mathcal{Y}, k)$ is nonempty for a class $k$. Then there exists a unique $j \in \mathcal{I}$ such that $k=k_{j}$ and

$$
\operatorname{DT}(\mathcal{Y}, k)=\operatorname{DT}\left(\left(\widehat{\mathcal{Y}}_{j}, c_{j}\right), k_{j}\right)
$$

Proof. First note that all the moduli stacks involved in Proposition 3.4.1 are Artin stacks locally of finite type by [17, Corollary 2.27], and hence they carry well defined Behrend's functions satisfying (iii) in [10, Theorem 4.3] by [10, Proposition 4.4, Corollary 4.5]. Moreover all these Artin stacks have affine geometric stabilizers by the discussions in [10, Section 5.1], and hence $\chi^{n a}$ can be defined on them ([10, Definition 2.3]). Now both claims follow by taking weighted Euler characteristics from both sides of the isomorphisms in Proposition 3.4.1.

Remark 3.4.4.

(1) It is interesting to note that Proposition 3.4.3 says that a counting invariant of stable sheaves on a gerbe $\mathcal{Y}$ coincides with a counting invariant of stable twisted sheaves on only one component of the dual $\widehat{\mathcal{Y}}$. Perhaps counting invariants of semistable sheaves on $\mathcal{Y}$ receives contributions from more than one component of $\widehat{\mathcal{Y}}$. We hope to study this in the future.

(2) In a similar fashion, one can prove a decomposition statement for the moduli spaces of torsion free sheaves with fixed determinants. This can be achieved by noting that the inverse functor to (3.1) takes the fixed determinant twisted sheaves on $\widehat{\mathcal{Y}}_{i}$ to the fixed determinant sheaves on $\mathcal{Y}$ (see [19, Section 7]).

(3) It might be more natural to study the decompositions of the counting invariants of $\mathcal{Y}$ with respect to the more general Bridgeland stability conditions. However, a theory of counting invariants with respect to Bridgeland stability conditions is currently not available. We consider our discussions in this paper as the first step towards a more in-depth study of decompositions of counting invariants on gerbes, a project which we hope to return to in the near future. 


\section{ACKNOWLEDGMENTS}

We thank A. Caldararu and M. Lieblich for discussions on twisted sheaves and gerbes, and X. Tang for helpful comments. We also thank the referee for very helpful suggestions. The second author was supported in part by NSF grant DMS-0757722.

\section{REFERENCES}

[1] D. Abramovich, T. Graber, and A. Vistoli. Algebraic orbifold quantum products. In: Adem, A.; Morava, J.; Ruan, Y. Orbifolds in mathematics and physics (Madison, WI, 2001), 1-24, Contemp. Math., 310, Amer. Math. Soc., 2002. MR1950940 (2004c:14104)

[2] D. Abramovich, T. Graber, and A. Vistoli. Gromov-Witten theory of Deligne-Mumford stacks. Amer. J. Math., 130(5):1337-1398, 2008. MR2450211(2009k:14108)

[3] K. Behrend. Donaldson-Thomas type invariants via microlocal geometry. Ann. Math. (2), 170(3):1307-1338, 2009. MR2600874 (2011d:14098)

[4] K. Behrend and B. Fantechi. The intrinsic normal cone. Invent. Math., 128(1):45-88, 1997. MR1437495 (98e:14022)

[5] A. Caldararu. Derived categories of twisted sheaves on Calabi-Yau manifolds. Ph.D thesis, Cornell University, 2000. MR2700538

[6] W. Chen and Y. Ruan. Orbifold Gromov-Witten theory. In: Adem, A.; Morava, J.; Ruan, Y. Orbifolds in mathematics and physics (Madison, WI, 2001), 25-85, Contemp. Math., 310, Amer. Math. Soc., 2002. MR1950941 (2004k:53145)

[7] D. Edidin, B. Hassett, A. Kresch, and A. Vistoli. Brauer groups and quotient stacks. Amer. J. Math., 123(4):761-777, 2001. MR1844577(2002f:14002)

[8] S. Hellerman, A. Henriques, T. Pantev, E. Sharpe, and M. Ando. Cluster decomposition, T-duality, and gerby CFTs. Adv. Theor. Math. Phys., 11(5):751-818, 2007. MR2365496 (2008m:81168)

[9] L. Illusie. Complexe cotangent et déformations. I. Lecture Notes in Mathematics, Vol. 239. Springer-Verlag, Berlin, 1971. MR0491680 (58:10886a)

[10] D. Joyce and Y. Song. A theory of generalized Donaldson-Thomas invariants. To appear in Mem. Amer. Math. Soc., arXiv:0810.5645v4.

[11] A. Kresch. On the geometry of Deligne-Mumford stacks. In: Abramovich, D.; Bertram, A.; Katzarkov, L.; Pandharipande, R.; Thaddeus, M. Algebraic Geometry: Seattle 2005. Proc. Sympos. Pure Math., 80, Part 1, Amer. Math. Soc., Providence, RI, 2009, 259-271. MR2483938(2011c:14006)

[12] M. Lieblich. Moduli of twisted sheaves. Duke Math. J., 138(1):23-118, 2007. MR2309155 (2008d:14018)

[13] M. Lieblich. Twisted sheaves and the period-index problem. Compos. Math., 144(1):1-31, 2008. MR2388554(2009b:14033)

[14] D. Maulik, N. Nekrasov, A. Okounkov, and R. Pandharipande. Gromov-Witten theory and Donaldson-Thomas theory. I. Compos. Math., 142(5):1263-1285, 2006. MR2264664 (2007i:14061)

[15] D. Maulik, N. Nekrasov, A. Okounkov, and R. Pandharipande. Gromov-Witten theory and Donaldson-Thomas theory. II. Compos. Math., 142(5):1286-1304, 2006. MR2264665 (2007i:14062)

[16] F. Nironi. Grothendieck Duality for Projective Deligne-Mumford Stacks. arXiv:0811.1955.

[17] F. Nironi. Moduli Spaces of Semistable Sheaves on Projective Deligne-Mumford Stacks. arXiv:0811.1949v1.

[18] M. Olsson and J. Starr. Quot functors for Deligne-Mumford stacks. Comm. Algebra, 31(8):4069-4096, 2003. MR2007396 (2004i:14002)

[19] X. Tang and H.-H. Tseng. Duality theorems of étale gerbes on orbifolds. arXiv:1004.1376.

[20] R. P. Thomas. A holomorphic Casson invariant for Calabi-Yau 3-folds, and bundles on K3 fibrations. J. Differential Geom., 54(2):367-438, 2000. MR1818182 (2002b:14049)

[21] R. P. Thomas and D. Huybrechts. Deformation-obstruction theory for complexes via Atiyah and Kodaira-Spencer classes. Math. Ann., 346(3):545-569, 2010. MR2578562 (2011b:14030) 
Department of Mathematics, Imperial College London, South Kensington Campus, LONDON SW7 2AZ, UNITED KINGDOM

E-mail address: a.gholampour@imperial.ac.uk

Current address: Department of Mathematics, University of Maryland, 1301 Mathematics Building, College Park, Maryland 20742-4015

E-mail address: amingh@umd.edu

Department of Mathematics, Ohio State University, 100 Math Tower, 231 West 18th Avenue, Columbus, Ohio 43210

E-mail address: hhtseng@math.ohio-state.edu 